\title{
CONCENTRAÇÃO DA DEMANDA ENERGÉTICA DA INDÚSTRIA BRASILEIRA DE ALIMENTOS E BEBIDAS
}

Luiz Moreira Coelho Junior ${ }^{1}$, Filipe Vanderlei Alencar ${ }^{2}$, Edvaldo Pereira Santos Júnior $^{2}$, Helena Cristina de Carvalho Soares ${ }^{2}$, Maria Luíza Coelho Cavalcanti ${ }^{3}$

${ }^{1}$ Professor do Departamento de Engenharia de Energias Alternativas e Renováveis DEER da Universidade Federal da Paraíba - UFPB, luiz@cear.ufpb.br;

${ }^{2}$ Graduandos em Engenharia de Energias Renováveis da UFPB.

${ }^{3}$ Mestranda pelo Programa de Pós-Graduação em Energias Renováveis da UFPB

Recebido em: 06/04/2018 - Aprovado em: 10/06/2018 - Publicado em: 20/06/2018 DOI: 10.18677/EnciBio_2018A114

\begin{abstract}
RESUMO
A Indústria Brasileira de Alimentos e Bebidas (IBAB) apresenta características singulares destacando-a dos demais setores. No consumo de energia, por exemplo, a IBAB possui relevância significativa, tanto para a nação quanto para o setor industrial como um todo. As questões energéticas ganham cada vez mais espaço e a avaliação da estrutura de mercado se faz necessário para orientação de políticas. Logo, este trabalho analisou a concentração energética da indústria brasileira de alimentos e bebidas, de 1970 a 2016. Os dados foram extraídos da Empresa de Pesquisa Energética e os indicadores usados para determinar a concentração foram: Razão de Concentração [CR(t)], Índice de Herfindahl-Hirschman (HHI), Índice de Entropia de Theil (E), e Índice de Gini (G). Os resultados mostraram que durante o período analisado, a principal fonte energética da IBAB foi o bagaço de cana de açúcar. $\mathrm{O} C R(\mathrm{t})$ evidencia que o consumo energético da IBAB se concentra nas 4 principais fontes. $\mathrm{O} \mathrm{HHI}$ infere tendências de crescimento e uma elevada concentração, no período estudado. $\mathrm{O} \mathrm{E}$ legitima as análises do $\mathrm{HHI}$. O G admite uma desigualdade Forte a Muito Forte. Contudo, a IBAB possui altos níveis de concentração na demanda energética, impactando negativamente na competitividade das fontes de energia do setor.
\end{abstract}

PALAVRAS-CHAVE: economia da energia, indicadores de concentração, matriz energética.

\section{ENERGY DEMAND CONCENTRATION OF THE BRAZILIAN FOOD AND BEVERAGE INDUSTRY}

\begin{abstract}
The Brazilian Food and Beverage industry (IBAB) presents unique characteristics highlighting it from other sectors. In energy consumption, for example, IBAB has significant relevance, both for the nation and for the industrial sector as a whole. Energy issues are gaining more and more space and the assessment of market structure is necessary for policy orientation. Therefore, this work analyzed the energy concentration of the Brazilian food and beverage industry from 1970 to 2016. Data were obtained from the Brazilian Energy Research Company and the indicators used to determine the concentration were: Concentration Ratio [CR (t)], Herfindahl-
\end{abstract}


Hirschman Index $(\mathrm{HHI})$, Theil Entropy Index $(\mathrm{E})$, and Gini Index $(\mathrm{G})$. The results showed that during the analyzed period, IBAB's main energy source was sugarcane bagasse. The CR $(\mathrm{t})$ shows that IBAB's energy consumption is concentrated in the 4 main sources. The $\mathrm{HHI}$ infers growth trends and a high concentration during the studied period. E legitimizes $\mathrm{HHI}$ analyzes. G admits a Strong to Very Strong inequality. However, IBAB has high levels of concentration in energy demand, negatively impacting the competitiveness of energy sources in the sector.

KEYWORDS: energy economy, energy matrix, concentration indicators.

\section{INTRODUÇÃO}

A Indústria Brasileira de Alimentos e Bebidas (IBAB) apresenta características singulares, destacando-a dos demais setores. O subsetor de bebidas possui tecnologias mais maduras e o subsetor de alimentos é um dos segmentos mais dinâmicos. Ambos atendem a demanda doméstica e externa (CERVIERI JÚNIOR, 2017; AMARAL; GUIMARÃES, 2017). Em 2016, a IBAB representou 10,1\% do PIB nacional e com faturamento líquido de $R \$ 614,3$ bilhões (ABIA, 2018). Este setor consumiu 23.531 mil toneladas equivalentes de petróleo (tep), representando $27,95 \%$ do segmento industrial e $9,78 \%$ do consumo energético nacional $74,47 \%$. Tendo o bagaço da cana de açúcar como principal fonte de energia, representando $74,47 \%$ da matriz energética da IBAB (EPE, 2018).

A dependência da IBAB com o bagaço da cana de açúcar promove discussão das questões energéticas no setor. Silva e Rodrigues (2015) avaliaram que a segurança energética busca, em parte, a diversificação. Nesse contexto, Coelho Junior et al. (2017) afirmaram que o excessivo poder de mercado, ou a não diversificação pode ocasionar uma alta dependência de determinado insumo energético.

A verificação da existência de poder de mercado no segmento energético é feita através da avaliação do grau de concentração. Essa avaliação usa, como ferramentas, indicadores empíricos estabelecidos na literatura (LUFT; ZILLI, 2013).Vários autores utilizaram os índices de concentração para analisar mercado em diversas áreas, por exemplo: no setor agrícola com Coelho Junior (2013), Costa e Santana (2014) e Coelho Junior (2017). No setor florestal, Coelho Junior et al. (2013) e Coelho Junior (2016) e para energia Coelho Junior et al. (2017).

Visto que as questões energéticas ganham cada vez mais espaço e compreender a estrutura do mercado seja imprescindível para orientação de políticas públicas. Por este motivo, este trabalho analisou a concentração da demanda energética da indústria brasileira de alimentos e bebidas, no período de 1970 a 2016.

\section{Dados utilizados}

\section{MATERIAL E MÉTODOS}

Os dados utilizados para verificar o grau de concentração da demanda de energia, em toneladas de petróleo equivalentes (tep), da indústria brasileira de alimentos e bebidas (IBAB) foram extraídos do Balanço Nacional de Energia (BEN), no período de 1970 a 2016.

\section{Taxa de crescimento geométrica (TCG)}

Cuenca e Dompieri (2017) sugeriram a Taxa de Crescimento Geométrica (TCG) como ferramenta para avaliar os acréscimos e decréscimos do consumo da $I B A B$, em taxas anuais, conforme a equação a seguir: 


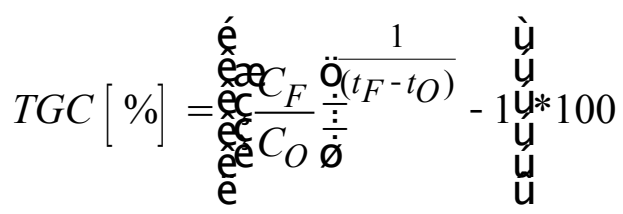

$\mathrm{O}$ consumo de energia $\left(\mathrm{C}_{\mathrm{F}}=\right.$ consumo no ano final e $\mathrm{C}_{\mathrm{O}}=$ consumo no ano inicial). Já os períodos $\left(t_{\mathrm{F}}=\right.$ último ano de análise e $t_{\mathrm{o}}=$ ano inicial de referência).

\section{Medidas de concentração}

O grau de concentração energética da indústria brasileira de alimentos e bebidas usou medidas de concentração, com base em Coelho Junior et al. (2013). Foram usados os seguintes indicadores de concentração: Razão de Concentração $[\mathrm{CR}(\mathrm{t})]$, Índice de Herfindahl-Hirschman $(\mathrm{HHI})$, Índice de Entropia de Theil $(\mathrm{E}) \mathrm{e}$ Coeficiente de Gini $(G)$.

Estes índices de concentração avaliam a estrutura de mercado por meio de um único indicador, pois se trata de um conceito de múltiplas dimensões. Para uma verificação mais precisa e robusta foram utilizados mais de um indicador, para que um complemente o outro auxiliando, na tomada de decisão conforme Coelho Junior et al. (2013).

\section{RESULTADOS E DISCUSSÃO}

A Tabela 1 revela o progresso do consumo de energia da indústria brasileira de alimentos e bebidas (IBAB) no período de 1970 até 2016. Ao longo de todo o período em estudo, a IBAB utilizou até 10 fontes na sua matriz energética. As principais foram: bagaço de cana de açucar, eletricidade e lenha. A IBAB consumiu, em 1970, 5,71 milhões tep, já em 2016 esse valor atingiu 23,53 milhões tep, com um TCG, entre esses anos, de 3,13\% a.a. O consumo acumulado total, entre 1970 e 2016 , foi de 605,99 milhões tep.

TABELA 1 Progresso da demanda energética da indústria brasileira de alimentos e bebidas, em $10^{3}$ tep, de 1970 a 2016.

\begin{tabular}{lrrrrrrrrrr}
\hline FONTES & $\mathbf{1 9 7 0}$ & $\mathbf{1 9 7 5}$ & $\mathbf{1 9 8 0}$ & $\mathbf{1 9 8 5}$ & $\mathbf{1 9 9 0}$ & $\mathbf{1 9 9 5}$ & $\mathbf{2 0 0 0}$ & $\mathbf{2 0 0 5}$ & $\mathbf{2 0 1 0}$ & $\mathbf{2 0 1 6}$ \\
\hline Gás natural & 0 & 0 & 0 & 9 & 131 & 164 & 226 & 511 & 662 & 833 \\
Carvão vapor & 0 & 0 & 63 & 108 & 108 & 89 & 49 & 62 & 71 & 51 \\
Lenha & 1.812 & 1.456 & 1.195 & 2.179 & 1.965 & 1.764 & 1.853 & 1.813 & 2.267 & 2.150 \\
Bagaç. de cana & 3.060 & 3.581 & 4.782 & 4.943 & 4.465 & 7.115 & 7.834 & 13.050 & 17.248 & 17.524 \\
Óleo diesel & 58 & 62 & 67 & 47 & 19 & 34 & 38 & 61 & 148 & 242 \\
Óleo combust. & 614 & 1.119 & 1.446 & 583 & 729 & 976 & 1.024 & 529 & 325 & 87 \\
Gás liq. & 4 & 9 & 14 & 14 & 18 & 33 & 97 & 125 & 202 & 331 \\
Petróleo & 9 & 14 & 15 & 8 & 8 & 6 & 2 & 0 & 0 & 0 \\
Querosene & 1 & 4 & 11 & 14 & 13 & 1 & 0 & 0 & 0 & 0 \\
Gás canalizado & 151 & 316 & 540 & 834 & 888 & 1.094 & 1.390 & 1.777 & 2.319 & 2.314 \\
Eletricidade & $\mathbf{5 . 7 1 0}$ & $\mathbf{6 . 5 6 2}$ & $\mathbf{8 . 1 3 2}$ & $\mathbf{8 . 7 3 8}$ & $\mathbf{8 . 3 4 6}$ & $\mathbf{1 1 . 2 7 6}$ & $\mathbf{1 2 . 5 1 4}$ & $\mathbf{1 7 . 9 2 6}$ & $\mathbf{2 3 . 2 4 4}$ & $\mathbf{2 3 . 5 3 1}$ \\
\hline TOTAL & & & & & & & & & &
\end{tabular}

Entre 1970 e 2016, a IBAB apresentou em média 23,43\% do consumo energético da Indústria nacional e da matriz energética brasileira foi de $8,86 \%$. $O$ bagaço de cana de açúcar liderou o ranking das fontes da IBAB. Em 1970, o consumo do bagaço de cana-de-açúcar representou $53,59 \%$ da demanda da IBAB e em 2016 cresceu para $74,47 \%$ do setor, apresentando um crescimento médio anual de 3,87\% a.a.. Rocha et al. (2010) afirmam que a maioria do bagaço de cana foi 
consumido na indústria de açúcar e destinado aos processos para produzir calor. De acordo com Oliveira et al. (2017), o bagaço de cana de açúcar vem do subproduto da produção sucroalcooleira e utilizada como fonte de energia em ciclos de cogeração de energia, aumentando a eficiência energética dessa indústria.

A eletricidade despontou grande relevância para a IBAB. Em 1970, a IBAB consumiu 0,15 milhões tep de eletricidade, já em 2016 foram consumidos 2,31 milhões tep, e teve o maior TCG $(0,37 \%$ a.a.) dentre as fontes para este período. Representando $9,83 \%$ do consumo da IBAB no ano de 2016. A lenha também tem grande importância para IBAB. Em 1970, consumiu 1,81 milhões tep e para 2016 demandaram 2,15 milhões tep de lenha, apresentando um crescimento médio anual de $0,37 \%$ a.a. e representou $9,13 \%$ do consumo da matriz energética da IBAB.

As demais fontes não apresentaram participação relevante. No período estudado a média da participação, destas demais fontes (todas excluindo o bagaço de cana, eletricidade e lenha), foram de 11,35\%, porém em 2016 representaram somente $6,56 \%$ do consumo total.

A Figura 1 mostra a evolução dos índices de concentração energética entre 1970 até 2016. A Figura 1(a) expõe a Razão Concentração [CR (t)] do consumo energético da indústria brasileira de alimentos e bebidas. De acordo com $\mathrm{CR}(\mathrm{t})$, a IBAB possui concentração nas quatro principais fontes da matriz energética. $O$ Quadro 1 auxiliou nas análises do CR(1), CR(2) e CR(4) uma vez que discrimina as quatro principais fontes para os anos de 1970, 1975, 1980, 1985, 1990, 1995, 2000, 2005, 2010 e 2016.

QUADRO 1 Fontes dominantes $\left(1^{\circ}, 2^{\circ}, 3^{\circ}\right.$ e $\left.4^{\circ}\right)$ de acordo com sua participação para indústria química brasileira 1970 a 2016.

\begin{tabular}{lcccc}
\hline Ano & $\mathbf{S}_{\mathbf{i}}\left(\mathbf{1}^{\circ}\right)$ & $\mathbf{S}_{\mathbf{i}}\left(\mathbf{2}^{\circ}\right)$ & $\mathbf{S}_{\mathbf{i}}\left(\mathbf{3}^{\circ}\right)$ & $\mathbf{S}_{\mathbf{i}}\left(\mathbf{4}^{\circ}\right)$ \\
\hline 1970 & Bagaço Cana & Lenha & Óleo Combust. & Eletricidade \\
1975 & Bagaço Cana & Lenha & Óleo Combust. & Eletricidade \\
1980 & Bagaço Cana & Óleo Combust. & Lenha & Eletricidade \\
1985 & Bagaço Cana & Lenha & Eletricidade & Óleo Combust. \\
1990 & Bagaço Cana & Lenha & Eletricidade & Óleo Combust. \\
1995 & Bagaço Cana & Lenha & Eletricidade & Óleo Combust. \\
2000 & Bagaço Cana & Lenha & Eletricidade & Óleo Combust. \\
2005 & Bagaço Cana & Lenha & Eletricidade & Óleo Combust. \\
2010 & Bagaço Cana & Eletricidade & Lenha & Gás Nat. \\
2016 & Bagaço Cana & Eletricidade & Lenha & Gás Nat. \\
\hline
\end{tabular}

Fonte: EPE (2018)

O $\mathrm{CR}(1)$ expõe que o setor de alimentos e bebidas é concentrado na maior fonte de energia. Entre 1970 e 2016, a média do $\operatorname{CR}(1)$ foi de $63,69 \%$. No ano de 2007 obteve-se a maior concentração, com 75,80\%, e em 1989 obteve-se a menor concentração, com 52,20\%. Durante todo o período estudado, de 1970 até 2016, a fonte com maior participação foi o bagaço da cana de açúcar.

De 1970 a 1990 o CR(1) apresentou comportamento de estabilidade, saindo de $53,58 \%$ para $53,51 \%$ respectivamente, com uma média de $\mathrm{CR}(1)$ de $57,20 \%$. Porém entre 1991 até 2016 houve tendência a crescimento. O CR(1) cresceu de 52,97\% (1991) para $74,47 \%$ (2016), com média de $68,94 \%$ para esse intervalo.

A razão de concentração para as duas maiores fontes $\mathrm{CR}(2)$ mostra que o consumo energético da IBAB é concentrado. De 1970 até 2016 a média do $\mathrm{CR}(2)$ foi $80,55 \%$. A maior concentração para duas fontes registrada foi em 1970 (85,33\%), enquanto que a menor foi em 1979 (76,16\%). As segundas principais fontes de 
energia, Si $\left(2^{\circ}\right)$, foram a Lenha $(1970$ - 1976, 1981 - 2005, 2008 e 2009) e a Eletricidade (2006, 2007 e 2010 - 2016) e Óleo Combustível (1977 - 1980).

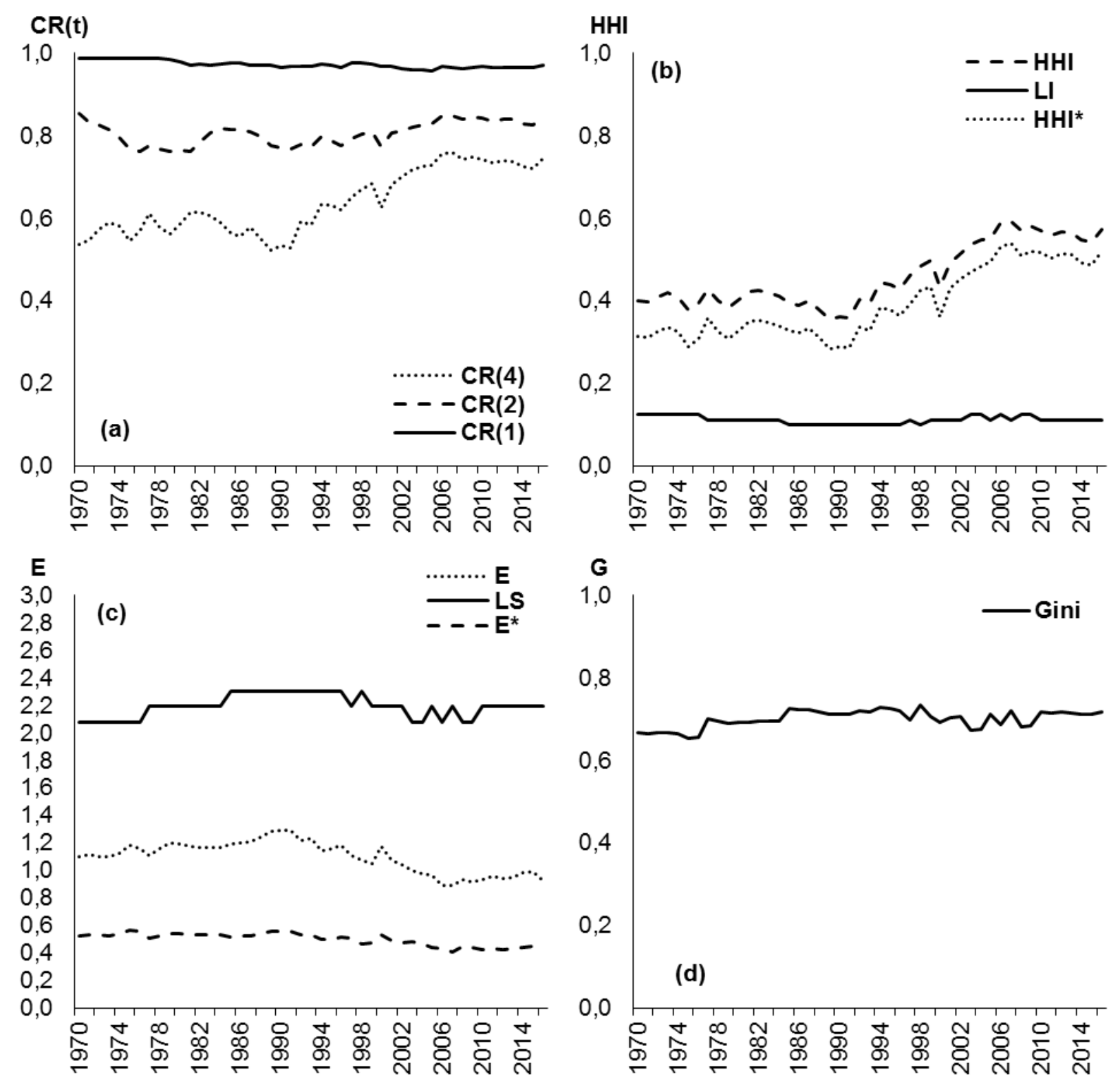

FIGURA 1 Evolução dos índices de concentração energética entre 1970 até 2016.

(a) Razão de concentração (b) Índice Herfindahl-Hirschman (c) Índice de entropia de Theil (d) Índice de Gini.

Em todo o período estudado o $\mathrm{CR}(2)$ manteve-se estável, com algumas oscilações devido às substituições das fontes energéticas. Entre 1976 e 1977 foi registrada a alocação da Lenha por Óleo Combustível como segunda maior fonte e, em 1980 e 1981 voltou a ser a Lenha. A partir de 2006 iniciou-se a substituição da Lenha pela Eletricidade como $\mathrm{Si}\left(2^{\circ}\right)$.

A avaliação do $\mathrm{CR}(4)$ inferiu uma alta concentração energética na IBAB entre os anos de 1970 até 2016. Neste período, o CR(4) obteve um média anual de $97,23 \%$, com máxima de $98,83 \%$ (1973) e mínimo de 97,23\% (2005). As terceiras, $\mathrm{Si}\left(3^{\circ}\right)$ principais fontes foram Óleo Combustível (1970 - 1976 e 1981 - 1983), Lenha (1977 - 1980, 2006, 20072010 - 2016) e Eletricidade (1984 - 2005, 2008 e 2009). 
As quartas, $\mathrm{Si}\left(4^{\circ}\right)$ principais fontes foram a Eletricidade (1970 - 1983), Óleo Combustível (1984 - 2005) e Gás Natural (2006 - 2016). A Figura 1 (b) mostra a evolução do índice de Herfindahl-Hirschman (HHI) da indústria brasileira de alimentos e bebidas (IBAB), entre 1970 até 2016. A diferença entre o HHI e LI $(\mathrm{HHI} / \mathrm{LI})$ e $\mathrm{HHI}{ }^{*}$ identifica quando a concentração da IBAB é muito alta, além disso, a concentração aumentou entre 1970 e 2016.

O HHI/LI apresentou comportamento de estabilidade entre 1970 e 1991, saindo de 0,2752 para 0,2579 e a média neste intervalo foi de 0,2858. Entre 1992 e 2016 houve um aumento da concentração energética da IBAB, proporcionado pelo incremento $\mathrm{HHI} / \mathrm{LI}$, que saiu de 0,3033 (1992) para 0,4631 (2016). O ano de maior concentração foi em $2007(0,4808)$ e em 1975 teve o menor registro $(0,2537)$.

$\mathrm{O} \mathrm{HHI}^{*}$, da mesma forma que o $\mathrm{HHI} / \mathrm{LI}$, apresentou estabilidade no comportamento no período de 1970 até 1991. Em 1970 o $\mathrm{HHI}^{*}$ foi avaliado em 0,3146 , enquanto que em 1991 valia 0,2866. No período de 1992 até 2016 , o $\mathrm{HHI}^{*}$ cresceu de 0,3370 para 0,5209, nessa ordem, indicando aumento da concentração nesse período. O ano com máxima concentração foi $2007, \mathrm{HHI}^{*}$ quantificado em 0,5408 , enquanto que o ano de mínima foi 1989 , o valor do $\mathrm{HH}^{*}$ foi 0,2847 . Uma vez que todos os valores do $\mathrm{HHI}^{*}$ foram maiores que 0,25 , então, este classificou a concentração da IBAB como muito alta em todo o período.

A Figura 1 (c) ilustra a mudança da concentração energética, através do Índice de Entropia de Theil (E), da IBAB entre 1970 e 2016. A diferença entre o LS e $E(E / L S)$ e $E^{*}$ mostram que a concentração energética da IBAB, entre 1970 e 2016, é alta, e tem tendência de crescimento.

O E/LS apresentou tendência de crescimento entre 1970 até 2016. Em 1970, o E/LS, foi quantificado em 0,9807, e cresceu para 1,2716, em 2016. Dessa forma, o aumento do E/LS representa aumento da concentração energética. Neste período, a média do E/LS foi 1,0967. O ano com maior concentração foi 2007 (1,3010), enquanto que $1991(0,6688)$ foi o ano com menor concentração.

$O E^{*}$ apresentou tendência de decrescimento de 0,5284 (1970) para 0,4213 (2016). Em conforme com o E/LS, a diminuição do $E^{*}$ representou o aumento da concentração energética entre 1970 e 2016. Durante este período, o $E^{*}$ apresentou média de 0,5004 . A maior concentração foi registrada em 2007 , o $E^{*}$ foi calculado em 0,4079, enquanto a menor concentração foi em 1975 ( $E^{*}$ avaliado em 0,4079).

A Figura 1(d) representa a evolução da concentração energética da IBAB, através do Índice de Gini (G), de 1970 a 2016. Este indicador mostrou que o mercado energético da IBAB possui uma classificação na desigualdade de forte a muito forte. Entre 1970 a 2016, o G apresentou tendências com baixo crescimento e algumas oscilações. Em 1970, a desigualdade marcou 0,6667, enquanto que em 2016 registrou-se 0,7186. Neste intervalo, o G teve média desigualdade foi, em 1983, com 0,7330 e a menor, em 1975, com 0,640. No período de 1970 até 1984, o $\mathrm{G}$ classificou a desigualdade energética da IBAB de Médio a Forte. Porém, com o crescimento do Gini, entre 1985 a 1996 a classificação passou a ser de Forte a Muito Forte. Entre 1997 e 2009, o Gini oscilou entre Médio a Forte e Forte a Muito Forte. De 2010 a 2016, o Gini retomou o crescimento e voltou a ser Forte a Muito Forte.

\section{CONCLUSÃO}

A partir das análises realizadas, conclui-se que: Durante o período analisado, a principal fonte energética da IBAB foi o bagaço de cana de açúcar. $O C R(t)$ evidencia que o consumo energético da IBAB se concentra nas 4 principais fontes. 
O HHI infere tendências de crescimento e uma elevada concentração, no período estudado. O $\mathrm{E}$ legitima as análises realizadas com $\circ \mathrm{HHI}$. $\mathrm{O} \mathrm{G}$ admite uma desigualdade Forte a Muito Forte. Contudo, a IBAB possui altos níveis de concentração na demanda energética, impactando negativamente na competitividade das fontes de energia do setor.

\section{REFERÊNCIAS}

AMARAL, G. F.; GUIMARÃES, D. D. Panoramas setoriais 2030: alimentos. In: Panoramas setoriais 2030: desafios e oportunidades para o Brasil. Rio de Janeiro, Banco Nacional de Desenvolvimento Econômico e Social, 2017. p. 59-68. Disponível em: <https://web.bndes.gov.br/bib/jspui/handle/1408/14234>. Acesso em: 9 de março de 2018

ABIA - Associação Brasileira da indústria de Alimentos. Faturamento. 2018. Disponível em: <https://www.abia.org.br/vsn/tmp_6.aspx?id=16>. Acesso em: 9 de março de 2018.

CERVIERI JÚNIOR, O. Panoramas setoriais 2030: bebidas. In: Panoramas setoriais 2030: desafios e oportunidades para o Brasil. Rio de Janeiro, Banco Nacional de Desenvolvimento Econômico e Social, 2017, p. 69-78.

COELHO JUNIOR, L. M. Concentração regional do valor bruto da produção do abacaxi no Paraná. Revista em Agronegócio e Meio Ambiente, v. 10, n. 4, p. 1015-1028, 2017. doi: <http://dx.doi.org/10.17765/2176-9168.2017v10n4p10151028>. Acesso em: 9 de março de 2018.

COELHO JUNIOR, L. M.. Concentração regional do valor bruto de produção da banana do Paraná, Brasil (1995 a 2010). Ciência Rural, v. 43, p. 2304-2310, 2013. doi: <http://dx.doi.org/10.1590/S0103-84782013005000137> . Acesso em: 22 de março de 2018.

COELHO JUNIOR, L. M.; ALENCAR, F. V.; SANTOS JUNIOR, E. P.; PINTO, P. A. L. A. Análise da concentração energética na indústria química brasileira. Enciclopédia biosfera, v. 14, p. 1332-1341, 2017. doi: <10.18677/EnciBio_2017B111 >. Acesso em: 9 de março de 2018.

COELHO JUNIOR, L. M.; DE REZENDE, J. L. P.; DE OLIVEIRA, A. D. Concentração das exportações mundiais de produtos florestais. Ciência Florestal, v. 23, n. 4, 2013. doi: <http://dx.doi.org/10.5902/1980509812353>. Acesso em: 14 de março de 2018.

COELHO JUNIOR, L. M.; REZENDE, J. L. P.; OLIVEIRA, A. D.. Concentração das exportações mundiais de produtos florestais. Ciência Florestal (UFSM. Impresso), v. 23, p. 691-701, 2013. Doi: <http://dx.doi.org/10.5902/1980509812353> Acesso em: 22 de março de 2018.

COELHO JUNIOR, M. L. Concentração regional do valor bruto de produção do pinhão no paraná. Ciência Florestal, v. 26, n. 3, p. 853-861, set. 2016. doi: < http://www.redalyc.org/html/534/53447685014/>. Acesso em: 9 de março de 2018. 
COSTA, N. L.; SANTANA, A. C. Estudo da concentração de mercado ao longo da cadeia produtiva da soja no Brasil. Revista de Estudos Sociais, v. 16, n. 32, p. 111135, 2014. doi: <http://dx.doi.org/10.19093/res.v16i32.1853>. Acesso em: 9 de março de 2018.

CUENCA, M. A. G.; DOMPIERI, M. H. G. Dinâmica espacial da canavicultura e análise dos efeitos sobre o valor bruto da produção, na região dos tabuleiros costeiros da Paraíba, Pernambuco e Alagoas. Revista Econômica do Nordeste, v. 47, n. 4, p. 91-106, 2017. Disponível em: <https://ren.emnuvens.com.br/ren/article/view/620/497>. Acesso em: 12 de março de 2018.

Empresa de Pesquisa Energética - EPE. Balanço energético nacional: séria histórica de 1970 até 2016. Capítulo 3: Consumo de energia por setor. 2018. Disponível em: <https://ben.epe.gov.br/BENSeriesCompletas.aspx >. Acesso em 9 de março de 2018.

LUFT, A.; ZILLI, J. B. Concentração de mercado: uma análise para a oferta de crédito pelo setor bancário brasileiro. Revista Teoria e Evidência Econômica, v. 19, n. 41, 2013. doi: <http://dx.doi.org/10.5335/rtee.v0i41.3737>. Acesso em: 9 de março de 2018.

OLIVEIRA, L. L.; NEVES, G. G.; WAITMAN, P. L. Estudo sobre cogeração de energia elétrica no setor sucroalcooleiro. REGRAD - Revista Eletrônica de Graduação do UNIVEM - ISSN 1984-7866, [S.I.], v. 10, n. 01, p. 354 - 365, oct. 2017. ISSN 1984-7866. Disponível em: <http://www.revista.univem.edu.br/REGRAD/article/view/2071>. Acesso em: 14 de março de 2018.

ROCHA, C. R.; BAJAY, S.; GORLA, F. D. Oportunidades de eficiência energética na indústria: relatório setorial do alimentos e bebidas. Brasília: CNI, 2010. Disponível em: $\quad$ <ttp://www.portaldaindustria.com.br/publicacoes/2012/9/oportunidades-deeficiencia-energetica-para-a-industria/>. Acesso em: 14 de março de 2018.

SILVA, A. C.; RODRIGUES, T. F. A segurança energética e um modelo para o futuro da Europa. Relações Internacionais, Lisboa, n. 46, p. 11-24, jun. 2015. doi: $<$ http://www.scielo.mec.pt/scielo.php?

script=sci_arttext\&pid=S164591992015000200002\&Ing=pt\&nrm=iso>. Acesso em: 9 de março de 2018. 